\title{
EPIDEMIOLOGY OF RARE DISEASES DETECTED BY NEWBORN SCREENING IN THE CZECH REPUBLIC
}

\author{
Jan David ${ }^{117}$, Petr Chrastina², Karolina Pešková2 ${ }^{2}$ Viktor Kožich², David Friedecký ${ }^{3}$ Tomáš Adam³ ${ }^{3}$ Eva Hlídková3, \\ Hana Vinohradská ${ }^{4}$, Dana Novotná ${ }^{5}$, Monika Hedelová', Eva Al Taji ${ }^{1}$, Andrea Holubová 6 , Veronika Skalická7, \\ Milan Macek ${ }^{6}$, Renata Gaillyová ${ }^{8}$, Felix Votava ${ }^{1}$ \\ 'Department of Children and Adolescents, Third Faculty of Medicine, Charles University and University Hospital Královské Vinohrady, Prague, \\ Czech Republic \\ ${ }^{2}$ Department of Paediatrics and Adolescent Medicine, First Faculty of Medicine, Charles University and General University Hospital, Prague, \\ Czech Republic \\ ${ }^{3}$ Department of Clinical Biochemistry, Faculty of Medicine, Palacký University and University Hospital Olomouc, Olomouc, Czech Republic \\ ${ }^{4}$ Department of Clinical Biochemistry, Faculty of Medicine, Masaryk University and University Hospital Brno, Brno, Czech Republic \\ ${ }^{5}$ Department of Paediatrics, Faculty of Medicine, Masaryk University and University Hospital Brno, Brno, Czech Republic \\ ${ }^{6}$ Department of Biology and Medical Genetics, Second Faculty of Medicine, Charles University and University Hospital Motol, Prague, Czech \\ Republic \\ ${ }^{7}$ Department of Paediatrics, Second Faculty of Medicine, Charles University and University Hospital Motol, Prague, Czech Republic \\ ${ }^{8}$ Department of Medical Genetics, Faculty of Medicine, Masaryk University and University Hospital Brno, Brno, Czech Republic
}

\section{SUMMARY}

Objectives: Presymptomatic detection of patients with rare diseases (RD), defined by a population frequency less than $1: 2,000$, is the task of newborn screening (NBS). In the Czech Republic (CZ), currently eighteen RD are screened: phenylketonuria/hyperphenylalaninemia (PKU/HPA), congenital hypothyroidism $(\mathrm{CH})$, congenital adrenal hyperplasia (CAH), cystic fibrosis (CF), medium chain acyl-CoA dehydrogenase deficiency (MCADD), long chain 3-hydroxyacyl-CoA dehydrogenase deficiency (LCHADD), very long chain acyl-CoA dehydrogenase deficiency (VLCADD), carnitine palmitoyl transferase I and II deficiency (CPTID, CPTIID), carnitine-acylcarnitine translocase deficiency (CACTD), maple syrup urine disease (MSUD), glutaric aciduria type I (GAI), isovaleryl-CoA dehydrogenase deficiency (IVA), argininemia (ARG), citrullinemia (CIT), biotinidase deficiency (BTD), cystathionine beta-synthase-deficient homocystinuria (CBSD HCU), and methylenetetrahydrofolate reductase deficiency homocystinuria (MTHFRD HCU). The aim was to analyze the prevalence of RD screened by NBS in CZ.

Methods: We examined the NBS programme in CZ from 1 January 2010 to 31 December 2017, which covered 888,891 neonates. Dried blood spots were primarily analyzed using fluorescence immuno-assay, tandem mass spectrometry and fluorimetry.

Results: The overall prevalence of RD among the neonate cohort was $1: 1,043$. Individually, $1: 2,877$ for $\mathrm{CH}, 1: 5,521$ for PKU/HPA, 1:6,536 for CF (1:5,887 including false negative patients), $1: 12,520$ for CAH, $1: 22,222$ for MCADD, $1: 80,808$ for LCHADD, 1:177,778 for GA I, 1: 177,778 for IVA, $1: 222,223$ for VLCADD, $1: 296,297$ for MSUD, $1: 8,638$ for BTD, and 1:181,396 for CBSD HCU.

Conclusions: The observed prevalence of RD, based on NBS, corresponds to that expected, more precisely it was higher for BTD and lower for MSUD, IVA, CBSD HCU, MCADD and VLCADD. Early detection of rare diseases by means of NBS is an effective secondary prevention tool.

Key words: rare disease, newborn screening, Czech Republic, public health, epidemiology, prevention

Address for correspondence: F. Votava, Department of Children and Adolescents, Third Faculty of Medicine, Charles University, University Hospital Královské Vinohrady, Šrobárova 1150/50, Prague 10, 10034 Prague, Czech Republic. E-mail: felix.votava@fnkv.cz

https://doi.org/10.21101/cejph.a5441

\section{INTRODUCTION}

Rare diseases (RD) are defined by a population frequency less than $1: 2,000$ and represent a heterogeneous group of up to 8,000 disorders (1). Despite significant therapeutic advances in the treatment of RD, many patients still suffer from insufficient diagnostics and inadequate care. With a view toward standardizing and harmonizing evidence-based health practices in the European Union, the Committee of Experts on RD (EUROCERD*) was

*www.eucerd.eu established in 2010 (2). The Government of the Czech Republic (CZ), through Resolution No. 466 of 14 June 2010, endorsed the "National Strategy for Rare Diseases for the Years 2010-2020" (3), which summarizes the issue of rare diseases from both the European Union and $\mathrm{CZ}$ viewpoints and proposes a core set of objectives and measures to improve RD diagnosis and treatment in $\mathrm{CZ}$. This Resolution includes newborn screening (NBS) as an important area since all diseases included in NBS are classified as RD and NBS represents a model approach to RD diagnosis and treatment.

NBS is an effective secondary prevention tool (4), for active population-wide detection of congenital and/or inherited 
diseases or defects in the early preclinical stages (5). The greater the number of neonates in a region that are screened, the greater the effectiveness of the NBS system. NBS is based on specific substances concentration measurement in dried blood spots (DBS) on filter paper and in selected probands on pathogenic allelic variants analysis in the same DBS. The overall NBS system is comprised of a reliable pre-analytical process (standardization of sample collection, timing, repeated samples, etc.), an analytical process (selection of laboratory methods, storage and utilization of samples), and a post-analytical process (protocols or procedures used in positive or unclear findings) (6). The rules of all these activities are summarized in the Methodological Guidelines Manual of the Czech Ministry of Health, which defines the medical lege artis procedures in the NBS including methods of diagnostic confirmation and subsequent care of patients detected by NBS (7).

NBS was first implemented in the CZ in 1975, for phenylketonuria/hyperphenylalaninemia (PKU/HPA) (8). NBS then expanded, in 1985 it was used for congenital hypothyroidism (CH) (9), in 2006 for 21-hydroxylase deficiency (congenital adrenal hyperplasia, CAH) (10), in 2009 for cystic fibrosis (CF) (11), and nine other inherited metabolic disorders (IMD), i.e., medium chain acyl-CoA dehydrogenase deficiency (MCADD), long chain 3-hydroxyacyl-CoA dehydrogenase deficiency (LCHADD), very long chain acyl-CoA dehydrogenase deficiency (VLCADD), carnitine palmitoyl transferase I and II deficiency (CPTID, CPTIID), carnitine-acylcarnitine translocase deficiency (CACTD), maple syrup urine disease (MSUD), glutaric aciduria, type I (GA I), and isovaleryl-CoA dehydrogenase deficiency (IVA) (12). The latest expansion was made in June 2016, when five additional IMD were added, i.e., argininemia (ARG), citrullinemia (CIT), biotinidase deficiency (BTD), cystathionine beta-synthase-deficient homocystinuria (CBSD HCU), and methylenetetrahydrofolate reductase deficiency homocystinuria (MTHFRD HCU).

The aim of this study was to analyze the epidemiology of RD screened by NBS in the CZ between years 2010 and 2017.

\section{MATERIALS AND METHODS}

The study (from 1 January 2010 to 31 December 2017) was based on results derived from DBS on filter paper that were collected from the heel pricks and sent to specified laboratories by mail (Table 1). DBS were taken between the 48th-72th hour of newborn's life. Our epidemiological data show the prevalence of screened RD at the time of DBS sampling.

The analysis included 888,891 neonates, which covers $100 \%$ of the Czech neonatal population (2010-2017). Data were collected from newborn screening laboratories (Table 1). DBS were tested for:
- thyroid-stimulating hormone (TSH), using fluorescence immuno-assay (FIA; Delfia a AutoDelfia produced by PerkinElmer) for detection of $\mathrm{CH}$ (13);

- 17-hydroxyprogesterone (17-OHP) for detection of CAH using the above-mentioned method (10);

- immunoreactive trypsinogen (IRT) for detection of CF using FIA, and from the group with the highest IRT levels, a subsequent DNA analysis of the CFTR (cystic fibrosis transmembrane conductance regulator) gene was performed (initially 32 and from July 2010, 50 common variants were tested using the commercial Elucigene assays produced by Elucigene Diagnostics) using the original DBS (11);

- amino acids and acylcarnitines for detection of IMD were determined using tandem mass spectrometry (MS/MS) using kits (MassChrom Reagent produced by Chromsystems) and MS/MS instrumentation (API 2000TM, API 3200TM and API 4000TM produced by AB Sciex), namely PKU/HPA, MCADD, LCHADD, VLCADD, CPTID, CPTIID, CACTD, MSUD, GA I, IVA, ARG, CIT, CBSD HCU, and MTHFRD HCU (14). Patients with BTD were detected using fluorimetry.

Newborns with positive NBS findings were referred for followup to appropriate clinical centres to confirm the diagnosis using generally accepted diagnostic standards: in cases with a confirmed diagnose patients started subsequent care (7). Decision limits for the screened disorders are summarized in Table 2 and confirmatory test criteria in Table 3 (15-19). The numbers of confirmed diagnoses from NBS were based on feedback reports from clinical care centres. In cases with unclear results (i.e. between negative and positive decision limits), disease specific protocols were applied (mostly repeated DBS sampling). The percentage of newborns with a final negative result was presented as the false positive rate (FPR). FPR was calculated as the ratio between the number of false positives and the total number of negatives findings. Positive predictive value (PPV) stated the probability that newborns with a positive screening test truly had the disease (the percentage of patients with a positive test who actually had the disease). PPV was calculated as the ratio between the number of true positives and the number of true positives and false positives findings).

\section{RESULTS}

The overall prevalence of RD among the neonate cohort was $1: 1,043$. Individually, $1: 2,877$ for $\mathrm{CH}, 1: 5,521$ for PKU/HPA, $1: 6,536$ for $\mathrm{CF}(1: 5,887$ including false negative patients), $1: 12,520$ for CAH, $1: 22,222$ for MCADD, $1: 80,808$ for LCHADD, $1: 177,778$ for GA I, $1: 177,778$ for IVA, $1: 222,223$ for VLCADD, $1: 296,297$ for MSUD, $1: 8,638$ for BTD, and $1: 181,396$ for CBSD HCU. Table 4 shows results of NBS in CZ from 2010-2017.

Table 1. Newborn screening laboratories in the Czech Republic

\begin{tabular}{|c|c|c|}
\hline Immunoanalytical methods & Tandem mass spectrometry, fluorimetry & DNA analysis \\
\hline $\begin{array}{c}\text { Department of Children and Adolescents, } \\
\text { University Hospital Královské Vinohrady, Prague }\end{array}$ & $\begin{array}{c}\text { Department of Paediatrics and Adolescent } \\
\text { Medicine, General University Hospital, Prague }\end{array}$ & $\begin{array}{c}\text { Department of Biology and Medical Genetics, } \\
\text { University Hospital Motol, Prague }\end{array}$ \\
\hline $\begin{array}{c}\text { Department of Clinical Biochemistry, University } \\
\text { Hospital Brno }\end{array}$ & $\begin{array}{c}\text { Department of Clinical Biochemistry, University } \\
\text { Hospital Olomouc }\end{array}$ & $\begin{array}{c}\text { Department of Medical Genetics, University } \\
\text { Hospital Brno }\end{array}$ \\
\hline
\end{tabular}


Table 2. Decision (positivity) limits for analytes detected in dried blood spots

\begin{tabular}{|c|c|c|}
\hline Disorder & Analyte & Decision limit (capillary blood) \\
\hline Congenital hypothyroidism & Thyroid-stimulating hormone (TSH) & $\mathrm{TSH} \geq 15.0 \mathrm{mlU} / \mathrm{L}$ \\
\hline Cystic fibrosis & $\begin{array}{l}\text { Immunoreactive trypsinogen (IRT) and CFTR } \\
\text { (cystic fibrosis transmembrane conductance } \\
\text { regulator) gene mutations }\end{array}$ & $\begin{array}{c}\mathrm{IRT}>99.0 \text { percentile }(65.0 \mathrm{ng} / \mathrm{mL}) \text { and } C F T R \\
\text { mutation on at least one allele or without mutation } \\
\text { and IRT } \geq 200 \mathrm{ng} / \mathrm{mL}\end{array}$ \\
\hline $\begin{array}{l}\text { Congenital adrenal hyperplasia } \\
\text { (21-hydroxylase deficiency) }\end{array}$ & 17-hydroxyprogesterone (17-OHP) & $\begin{array}{l}\text { 17-OHP according to birthweight/gestational age, } \\
\quad \text { range } \\
\begin{array}{l}20.0-160 \mathrm{nmol} / \mathrm{L}, \text { example: } 20.0 \mathrm{nmol} / \mathrm{L} \text { for } \\
\geq 2700 \mathrm{~g}(\geq 37 \text { gestational week })\end{array}\end{array}$ \\
\hline Phenylketonuria/hyperphenylalaninemia & $\begin{array}{c}\text { Phenylalanine (Phe) } \\
\text { Tyrosine (Tyr) }\end{array}$ & $\begin{array}{c}\text { Phe }>120 \mu \mathrm{mol} / \mathrm{L} \text { and Phe/Tyr } \\
\text { ratio }>2.00\end{array}$ \\
\hline Medium chain acyl-CoA dehydrogenase deficiency & $\begin{array}{l}\text { Octanoylcarnitine (C8) } \\
\text { Acetylcarnitine (C2) }\end{array}$ & $\begin{array}{c}\mathrm{C} 8>0.40(0.50) \mu \mathrm{mol} / \mathrm{L} \text { and } \mathrm{C} 8 / \mathrm{C} 2 \text { ratio } \\
>0.02(0.03) \mu \mathrm{mol} / \mathrm{L}\end{array}$ \\
\hline $\begin{array}{l}\text { Long chain 3-hydroxyacyl-CoA dehydrogenase } \\
\text { deficiency }\end{array}$ & $\begin{array}{l}\text { Hydroxypalmitoylcarnitine }(\mathrm{C} 16 \mathrm{OH}) \\
\text { Hydroxyoleoylcarnitine }(\mathrm{C} 18: 1 \mathrm{OH})\end{array}$ & $\begin{aligned} \mathrm{C} 16 \mathrm{OH} & >0.10 \mu \mathrm{mol} / \mathrm{L} \text { or } \mathrm{C} 18: 10 \mathrm{H} \\
& >0.10(0.07) \mu \mathrm{mol} / \mathrm{L}\end{aligned}$ \\
\hline $\begin{array}{l}\text { Very long chain acyl-CoA dehydrogenase } \\
\text { deficiency }\end{array}$ & $\begin{array}{c}\text { Tetradecenoylcarnitine (C14:1) } \\
\text { Acetylcarnitine (C2) } \\
\text { Palmitoylcarnitine (C16) }\end{array}$ & $\begin{array}{l}\qquad \mathrm{C} 14: 1>0.55(0.40) \mu \mathrm{mol} / \mathrm{L} \\
\text { and } \mathrm{C} 14: 1 / \mathrm{C} 2 \text { ratio }>0.03 \\
\text { and } \mathrm{C} 14: 1 / \mathrm{C} 16 \text { ratio }>0.26(0.15)\end{array}$ \\
\hline Carnitine palmitoyl transferase I deficiency & $\begin{array}{c}\text { Free carnitine }(\mathrm{C} 0) \\
\text { Palmitoylcarnitine }(\mathrm{C} 16) \\
\text { Oleoylcarnitine (C18:1) } \\
\text { Acetylcarnitine (C2) }\end{array}$ & $\begin{array}{l}\qquad \mathrm{C} 0>60.3(57.0) \mu \mathrm{mol} / \mathrm{L} \\
\text { and } \mathrm{C} 0 /(\mathrm{C} 16+\mathrm{C} 18) \text { ratio }>25.0(29.0) \\
\text { and }(\mathrm{C} 16+\mathrm{C} 18: 1) / \mathrm{C} 2 \text { ratio }<0.10\end{array}$ \\
\hline $\begin{array}{l}\text { Carnitine palmitoyl transferase II deficiency } \\
\text { Carnitine-acylcarnitine translocase deficiency }\end{array}$ & $\begin{array}{c}\text { Palmitoylcarnitine (C16) } \\
\text { Oleoylcarnitine (C18:1) } \\
\text { Acetylcarnitine (C2) } \\
\end{array}$ & $\begin{array}{c}\mathrm{C} 16>5.06(7.00) \mu \mathrm{mol} / \mathrm{L} \\
\text { and }(\mathrm{C} 16+\mathrm{C} 18: 1) / \mathrm{C} 2 \text { ratio }>0.35(0.48)\end{array}$ \\
\hline Glutaric aciduria type I & $\begin{array}{l}\text { Glutarylcarnitine (G5DC) } \\
\text { Octanoylcarnitine (C8) } \\
\text { Palmitoylcarnitine (C16) }\end{array}$ & $\begin{aligned} \mathrm{C} 5 \mathrm{DC}> & >0.40(0.60) \mu \mathrm{mol} / \mathrm{L} \\
\text { and } \mathrm{C} 5 \mathrm{DC} / \mathrm{C} 8 \text { ratio } & >5.40(\mathrm{C} 5 \mathrm{DC} / \mathrm{C} 16 \\
& >0.40)\end{aligned}$ \\
\hline Maple syrup urine disease & $\begin{array}{c}\text { Leucine (Leu) } \\
\text { Isoleucine (Isoleu) } \\
\text { Hydroxyproline (Hyp) } \\
\text { Alanine (Ala) } \\
\text { Valine (Val) } \\
\text { Tyrosine (Tyr) } \\
\text { Phenylalanine (Phe) }\end{array}$ & $\begin{aligned} \text { Leu + Isoleu }+ \text { Hyp }> & 270 \mu \mathrm{mol} / \mathrm{L}(\text { Leu }>260 \\
& \mu \mathrm{mol} / \mathrm{L}) \\
\text { and Leu/Ala ratio } & >1.40(1.25) \text { or Leu + Val/Phe } \\
+ & \text { Tyr }>3.79\end{aligned}$ \\
\hline Isovaleryl-CoA dehydrogenase deficiency & $\begin{array}{c}\text { Isovalerylmethybutyrylcarnitine (C5) } \\
\text { Free carnitine (C0) } \\
\text { Propionylcarnitine (C3) } \\
\text { Octanoylcarnitine (C8) }\end{array}$ & $\begin{array}{c}\mathrm{C} 5>1.00(0.60) \mu \mathrm{mol} / \mathrm{L} \\
\text { and } \mathrm{C} 5 / \mathrm{C} 0 \text { ratio }>0.03 \\
\text { and } \mathrm{C} 5 / \mathrm{C} 3 \text { ratio }>0.39(\mathrm{C} 5 / \mathrm{C} 8 \text { ratio }>20.0)\end{array}$ \\
\hline Citrullinemia & $\begin{array}{c}\text { Citrulline (Cit) } \\
\text { Ornithine (Orn) } \\
\text { Phenylalanine (Phe) }\end{array}$ & $\begin{array}{l}\text { Cit }>70.0(56.0) \mu \mathrm{mo} / \mathrm{L} \\
\text { and Orn/Cit ratio }<2.09(2.51) \\
\text { and Cit/Phe ratio }>0.95(0.81)\end{array}$ \\
\hline Biotinidase deficiency & Biotinidase serum activity & $\begin{array}{c}\text { Biotinidase serum activity }<30.0 \% \text { than median of } \\
\text { health population }\end{array}$ \\
\hline $\begin{array}{c}\text { Cystathionine beta-synthase-deficient homo- } \\
\text { cystinuria }\end{array}$ & $\begin{array}{l}\text { Methionine (Met) } \\
\text { Phenylalanine (Phe) } \\
\text { Homocystein (Hcys) }\end{array}$ & $\begin{array}{l}\quad \text { Met }>33.0(36.4) \mu \mathrm{mol} / \mathrm{L} \\
\text { and Met/Phe ratio }>0.58(0.41) \\
\text { and Hcys }>12.0(15.0) \mu \mathrm{mol} / \mathrm{L}\end{array}$ \\
\hline $\begin{array}{l}\text { Methylenetetrahydrofolate reductase deficiency } \\
\text { homocystinuria }\end{array}$ & $\begin{array}{c}\text { Methionine (Met) } \\
\text { Phenylalanine (Phe) } \\
\text { Homocystein (Hcys) }\end{array}$ & $\begin{array}{c}\text { Met }<7.00 \mu \mathrm{mol} / \mathrm{L} \text { or Met/Phe ratio }<0.15(0.10) \\
\text { and Hcys }>12.00(15.0) \mu \mathrm{mol} / \mathrm{L}\end{array}$ \\
\hline Argininemia & $\begin{array}{c}\text { Arginine (Arg) } \\
\text { Ornithine (Orn) } \\
\text { Phenylalanine (Phe) }\end{array}$ & $\begin{array}{c}\quad \operatorname{Arg}>60.0(63.0) \mu \mathrm{mol} / \mathrm{L} \\
\text { and Arg/Orn ratio }>0.75(0.40) \\
\text { and Arg/Phe ratio }>1.02(0.98)\end{array}$ \\
\hline
\end{tabular}

Decision limits for amino acids and acylcarnitines in parentheses are for non-derivatized assays. 
Table 3. Confirmatory test criteria for screened disorders

\begin{tabular}{|c|c|}
\hline Disorder & Definition (venous blood sample) \\
\hline Congenital hypothyroidism & Thyroid-stimulating hormone $(\mathrm{TSH})>8.00 \mathrm{mlU} / \mathrm{L}$ or free thyroxine $(\mathrm{fT} 4)<12.0 \mathrm{pmol} / \mathrm{L}$ \\
\hline Cystic fibrosis & $\begin{array}{l}\text { Sweat test } \geq 60.0 \mathrm{mmol} / \mathrm{L} \text { or } 30.0-59.0 \mathrm{mmol} / \mathrm{L} \text { and two pathogenic mutations in } \\
\text { cystic fibrosis transmembrane conductance regulator }(C F T R) \text { gene }\end{array}$ \\
\hline $\begin{array}{l}\text { Congenital adrenal hyperplasia } \\
\text { (21-hydroxylase deficiency) }\end{array}$ & $\begin{array}{c}\text { Basal level of 17-hydroxyprogesterone (17-OHP) above reference range and/or posi- } \\
\text { tive cosyntropin test and casual mutation in CYP21A2 gene }\end{array}$ \\
\hline Phenylketonuria/hyperphenylalaninemia & Phenylalanine $(\mathrm{Phe})>120 \mu \mathrm{mol} / \mathrm{L}$ \\
\hline Medium chain acyl-CoA dehydrogenase (MCAD) deficiency & $\begin{array}{l}\text { Octanoylcarnitine (C8) and acetylcarnitine }(\mathrm{C} 2) \text { above reference range, and MCAD } \\
\text { deficiency or two pathogenic mutations in ACADM gene or decreased fatty acid oxida- } \\
\text { tion (FAO) in lymphocytes }\end{array}$ \\
\hline Long chain 3-hydroxyacyl-CoA dehydrogenase (LCHAD) deficiency & $\begin{array}{l}\text { Hydroxypalmitoylcarnitine }(\mathrm{C} 16 \mathrm{OH}) \text { and hydroxyoleoylcarnitine }(\mathrm{C} 18: 10 \mathrm{H}) \text { above } \\
\text { reference range, and } \mathrm{LCHAD} \text { deficiency or two pathogenic mutations in } \mathrm{HADHA} \text { gene } \\
\text { or decreased FAO in lymphocytes }\end{array}$ \\
\hline Very long chain acyl-CoA dehydrogenase (VLCAD) deficiency & $\begin{array}{l}\text { Tetradecenoylcarnitine (C14:1) and acylcarnitine }(\mathrm{C} 2) \text { and palmitoylcarnitine }(\mathrm{C} 16) \\
\text { above reference range and VLCAD deficiency or two pathogenic mutations in } \\
\text { ACADVL gene or decreased FAO in lymphocytes }\end{array}$ \\
\hline Carnitine palmitoyl transferase (CPT) I deficiency & $\begin{array}{c}\text { Free carnitine }(\mathrm{C} 0) \text { and palmitoylcarnitine }(\mathrm{C} 16) \text { and oleoylcarnitine }(\mathrm{C} 18: 1) \text { and } \\
\text { acetylcarnitine }(\mathrm{C} 2) \text { above reference range and } \mathrm{CPT} \text { I deficiency or two pathogenic } \\
\text { mutations in CPT IA gene or decreased FAO in lymphocytes }\end{array}$ \\
\hline Carnitine palmitoyl transferase (CPT) II deficiency & $\begin{array}{l}\text { Palmitoylcarnitine (C16) and oleoylcarnitine (C18:1) and acetylcarnitine (C2) above } \\
\text { reference range, and CPT II deficiency or two pathogenic mutations in CPT2 gene }\end{array}$ \\
\hline Carnitine-acylcarnitine translocase (CACT) deficiency & $\begin{array}{l}\text { Palmitoylcarnitine (C16) and oleoylcarnitine (C18:1) and acetylcarnitine (C2) above } \\
\text { reference range and CACT deficiency or two pathogenic mutations in SLC25A20 } \\
\text { gene }\end{array}$ \\
\hline Glutaric aciduria type I & $\begin{array}{c}\text { Glutarylcarnitine }(\mathrm{C} 5 \mathrm{DC}) \text { and octanoylcarnitine }(\mathrm{C} 8) \text { and palmitoylcarnitine }(\mathrm{C} 16) \\
\text { above reference range and glutaryl } \mathrm{CoA} \text { dehydrogenase deficiency or two pathogenic } \\
\text { mutations in } G C D \text { gene }\end{array}$ \\
\hline Maple syrup urine disease & $\begin{array}{c}\text { Leucine (Leu) and isoleucine (Isoleu) and hydroxyproline and alanine (Ala) and } \\
\text { valine (Val) and tyrosine (Tyr) and phenylalanine (Phe) above reference range and } \\
\text { branched-chain ketoacid dehydrogenase (BCKAD) deficiency or two pathogenic } \\
\text { mutations in BCKDHA gene or BCKDHB gene or DBT gene }\end{array}$ \\
\hline Isovaleryl-CoA dehydrogenase deficiency & $\begin{array}{l}\text { Isovalerylmethybutyrylcarnitine (C5) and free carnitine }(\mathrm{CO}) \text { and propionylcarnitine } \\
\text { (C3) and octanoylcarnitine (C8) above reference range and isovaleryl CoA dehydro- } \\
\text { genase deficiency or two pathogenic mutations in IVD gene }\end{array}$ \\
\hline Citrullinemia & Argininosuccinate synthase deficiency or two pathogenic mutations in ASS1 gene \\
\hline Biotinidase deficiency & Biotinidase deficiency or two pathogenic mutations in BTD gene \\
\hline Cystathionine beta-synthase-deficient homocystinuria & Cystathionine beta-synthase deficiency or two pathogenic mutations in CBS gene \\
\hline Methylenetetrahydrofolate reductase deficiency homocystinuria & $\begin{array}{l}\text { Methylenetetrahydrofolate reductase deficiency or two pathogenic mutations in } \\
\text { MTHFR gene }\end{array}$ \\
\hline Argininemia & Arginase deficiency or two pathogenic mutations in $A R G 1$ gene \\
\hline
\end{tabular}

\section{DISCUSSION}

We have quantified prevalence at the age of DBS sampling. Considering that screened RD are inherited conditions and thanks to NBS can be treated in a timely manner, and based on a normal life expectancy during childhood, it can be assumed that the prevalence of RD is almost the same as their frequency and incidence in the general paediatric population. Table 5 summarizes the data from the literature of other countries relative to the prevalence of RD screened in CZ (18, 20-22). A comparison with our results leads us to conclude that the prevalence in the Czech population is higher for BTD, but lower for MSUD, IVA, CBSD HCU, MCADD, and VLCADD. However, in case of low prevalence, the statistical effect of small numbers may occur.
One interesting result was the markedly lower population frequency of $\mathrm{CF}$ than reported previously, i.e. $1: 2,700$ based on clinical observations in CZ (23). The explanation for this difference likely rests with the increasing effect of prenatal diagnosis and better-informed reproductive decisions (22).

The cause of false negativity in CF NBS is predominantly due to lower IRT levels in CF newborns with meconium ileus (6 cases from 15 false negative patients). While these infants can be detected clinically, there are nevertheless a small number of CF newborns that escape detection due to very rare mutations on both alleles.

The improving efficacy of NBS to detect RD in CZ was also documented by the increasing cumulative screening prevalence with the stepwise expansion of screened disorders from $1: 2,701$ 
Table 4. Results of newborn screening in the Czech Republic from 2010-2017 (N=888,891)

\begin{tabular}{|c|c|c|c|c|c|c|c|}
\hline Disease & Period & $\begin{array}{c}\text { Number of } \\
\text { screened } \\
\text { neonates }(n)\end{array}$ & $\begin{array}{l}\text { Number of } \\
\text { confirmed } \\
\text { diagnosis (n) }\end{array}$ & $\begin{array}{l}\text { Screening } \\
\text { prevalence }\end{array}$ & $\begin{array}{l}\text { Number of FP } \\
\text { (n) }\end{array}$ & $\begin{array}{l}\text { FPR total } \\
(\%)\end{array}$ & PPV \\
\hline PKU/HPA & \multirow{12}{*}{$\begin{array}{c}\operatorname{Jan} 1,2010-D e c \\
31,2017\end{array}$} & \multirow{12}{*}{888,891} & 161 & $1: 5,521$ & 238 & 0.0268 & 0.40 \\
\hline $\mathrm{CH}$ & & & 309 & $1: 2,877$ & 197 & 0.0222 & 0.61 \\
\hline $\mathrm{CAH}$ & & & 71 & $1: 12,520$ & 3696 & 0.4158 & 0.02 \\
\hline $\mathrm{CF}$ & & & 136 & $1: 6,536$ & 967 & 0.1088 & 0.12 \\
\hline MCADD & & & 40 & $1: 22,222$ & 17 & 0.0019 & 0.70 \\
\hline LCHADD & & & 11 & $1: 80,808$ & 4 & 0.0004 & 0.73 \\
\hline VLCADD & & & 4 & $1: 222,223$ & 62 & 0.0070 & 0.06 \\
\hline CPTID & & & 0 & - & 29 & 0.0033 & - \\
\hline CPTIID/CACTD & & & 0 & - & 2 & 0.0002 & - \\
\hline MSUD & & & 3 & $1: 296,297$ & 90 & 0.0101 & 0.03 \\
\hline GAI & & & 5 & 1:177,778 & 29 & 0.0033 & 0.15 \\
\hline IVA & & & 5 & 1:177,778 & 75 & 0.0084 & 0.06 \\
\hline ARG & \multirow{5}{*}{$\begin{array}{c}\text { Jun 1, 2016-Dec } \\
31,2017\end{array}$} & \multirow{5}{*}{181,396} & 0 & - & 1 & 0.0006 & - \\
\hline CIT & & & 0 & - & 10 & 0.0055 & - \\
\hline BTD & & & 21 & $1: 8,638$ & 34 & 0.0187 & 0.38 \\
\hline CBSD HCU & & & 1 & $1: 181,396$ & 10 & 0.0055 & 0.09 \\
\hline MTHFRD HCU & & & 0 & - & 3 & 0.0017 & - \\
\hline Total & & & 767 & $1: 1,043$ & 5,464 & 0.6387 & 0.12 \\
\hline
\end{tabular}

ARG - argininemia; BTD - biotinidase deficiency; CACTD - carnitine-acylcarnitine translocase deficiency; CAH - congenital adrenal hyperplasia; CBSD HCU - cystathionine beta-synthase-deficient homocystinuria; $\mathrm{CF}$ - cystic fibrosis; $\mathrm{CH}$ - congenital hypothyroidism; CIT - citrullinemia; CPTID - carnitine palmitoyl transferase I deficiency; CPTIID - carnitine palmitoyl transferase II deficiency; FP - false positivity; FPR - false positive rate; GA I - glutaric aciduria type I (glutaryl-CoA dehydrogenase deficiency); IVA - isovaleryl-CoA dehydrogenase deficiency (isovaleric acidaemia); LCHADD - long chain 3-hydroxyacyl-CoA dehydrogenase deficiency; MCADD - medium chain acyl-CoA dehydrogenase deficiency; MSUD - maple syrup urine disease; MTHFRD HCU - methylenetetrahydrofolate reductase deficiency homocystinuria; PKU/HPA phenylketonuria/hyperphenylalaninemia; PPV - positive predictive value; VLCADD - very long chain acyl-CoA dehydrogenase deficiency

Table 5. Literary data on prevalence of screened rare diseases (18, 20-22)

\begin{tabular}{|l|c|}
\hline Disease & Prevalence \\
\hline Congenital hypothyroidism & $1: 2,600$ \\
\hline Phenylketonuria/hyperphenylalaninemia & $1: 2,000-1: 10,000$ \\
\hline Cystic fibrosis & $1: 3,000-1: 13,500$ \\
\hline Congenital adrenal hyperplasia & $1: 14,000$ \\
\hline Medium chain acyl-CoA dehydrogenase deficiency & $1: 15,000$ \\
\hline Cystathionine beta-synthase-deficient homocystinuria & $1: 60,000$ \\
\hline Biotinidase deficiency & $1: 30,000-1: 60,000$ \\
\hline Citrullinemia & $1: 40,000$ \\
\hline Long chain 3-hydroxyacyl-CoA dehydrogenase deficiency & $1: 100,000$ \\
\hline Isovaleryl-CoA dehydrogenase deficiency (isovaleric acidaemia) & $1: 100,000$ \\
\hline Maple syrup urine disease & $1: 150,000$ \\
\hline Very long chain acyl-CoA dehydrogenase deficiency & $1: 11,000-1: 100,000$ \\
\hline Carnitine palmitoyl transferase II deficiency & $1: 100,000$ \\
\hline Argininemia & $1: 1,000,000$ \\
\hline Carnitine palmitoyl transferase I deficiency & $1: 1,000,000$ \\
\hline Carnitine-acylcarnitine translocase deficiency & $1: 1,000,000$ \\
\hline Glutaric aciduria type I (glutaryl-CoA dehydrogenase deficiency) & Unknown \\
\hline Methylenetetrahydrofolate reductase deficiency homocystinuria & Unknown \\
\hline
\end{tabular}


between 2002-2005 to $1: 2,072$ in 2007-2008 (24), to $1: 1,043$ in the currently evaluated period (2010-2017). In a comparison of the number of screened disorders in Europe (25), CZ ranks better than average. On the other hand, the achieved high NBS detection levels were associated with an increasing frequency of repeated DBS, with the current cumulative FPR $0.64 \%$. FPR impacts the healthy population and can stigmatize neonates and their families (26). Reducing the FPR is a challenge and one of the objectives of the NBS system. From our results, CAH has the highest FPR, with other screened disorders having significantly lower FPR. An effective way to reduce FPR is by implementation of a secondary analytic tier based on the original DBS: in case of CAH for example, using liquid chromatography with MS/MS (27). Pilot studies looking for ways to address this issue are in progress abroad and in CZ.

The greater number of diseases screened by the NBS system corresponds with technological progress and analytic potential. However, expanding the NBS system can create problems, not only technical, but also ethical, economic, legislative, and political ones. Current $\mathrm{Czech}$ legislation does not allow the nationwide NBS, which would be primarily based on genome analysis, e.g. NBS for spinal muscular atrophy, although it would be effective for early diagnosis and therapy (28).

The above-mentioned issues have led to discussions about adding or refining the original criteria of the NBS system, defined in 1968 by Wilson and Jungner (5). Every NBS expansion is associated with questions about the selection criteria. Traditional screening criteria can function as guidelines even if their universal applicability has been questioned by new biotechnologies and scientific progress. Before adding a new disorder to the screening panel, it is necessary to evaluate the balance between health benefits and potential harms (26). In 2010-2011, the European Network of Experts on Newborn Screening (EUNENBS) created a questionnaire study and published a list of 26 diseases, which

Table 6. Recommendation of diseases for newborn screening in Europe (29)

\begin{tabular}{|c|c|c|}
\hline \multicolumn{2}{|c|}{ Basic group } & \multirow[b]{2}{*}{ Candidate group } \\
\hline $\begin{array}{l}\text { Diseases with higher } \\
\text { prevalence }\end{array}$ & $\begin{array}{l}\text { Diseases with lower } \\
\text { prevalence }\end{array}$ & \\
\hline $\begin{array}{c}\text { PKU/HPA, CH, CAH, } \\
\text { CF, MCADD, Th }\end{array}$ & MSUD, GA I, GAL & $\begin{array}{c}\text { BD, CPTIID, CACTD, } \\
\text { GA II, HMGD, HCSD, } \\
\text { HCU, IVA, BKT, } \\
\text { LCHADD, LSD, 3MCC, } \\
\text { TYR I, TYR II, TYR III, } \\
\text { VLCADD, vitamin B12 } \\
\text { deficiency, SCID, CMV }\end{array}$ \\
\hline
\end{tabular}

3MCC - 3-methylcrotonyl-CoA carboxylase deficiency; BD - biotinidase deficiency; BKT - $\beta$-ketothiolase deficiency; CACTD - carnitine-acylcarnitine translocase deficiency; $\mathrm{CAH}$ - congenital adrenal hyperplasia; $\mathrm{CF}$ - cystic fibrosis; $\mathrm{CH}$ - congenital hypothyroidism; CMV - congenital cytomegalovirus infection; CPTIID - carnitine palmitoyl transferase II deficiency; HCU - homocystinuria; HCSD - holocarboxylase synthetase deficiency; HMGD - HMG-CoA lyase deficiency; GA I, II - glutaric aciduria type I, II; GAL - galactosemia; IVA - isovaleryl-CoA dehydrogenase deficiency; LCHADD - long chain 3-hydroxyacyl-CoA dehydrogenase deficiency; LSD - Iysosomal storage disorders; MCADD - medium chain acyl-CoA dehydrogenase deficiency; MSUD - maple syrup urine disease; PKU/HPA - phenylketonuria/hyperphenylalaninemia; SCID - severe combined immunodeficiency; Th - thalassemia; TYR I, II, III - tyrosinemia type I, II, III; VLCADD - very long chain acyl-CoA dehydrogenase deficiency could be included in European NBS system (29). The list is divided into basic groups with higher and lower prevalence and candidate groups (Table 6). In CZ, the study of NBS of severe combined immunodeficiency (SCID) has already been methodologically prepared (30).

\section{CONCLUSIONS}

The prevalence of screened RD in the Czech population mostly corresponds with internationally published data, actually, it was found higher for BTD and lower for MSUD, IVA, CBSD HCU, MCADD, and VLCADD.

NBS in CZ detects patients with RD in the early preclinical stages and the level of NBS corresponds with the standard used by many states of the European Union. NBS in CZ represents an efficient tool to improve the quality of care for patients with RD. The next important steps in NBS optimization will be to examine additional analytical methods to reduce false positivity, consider expanding the list of screened disorders and discuss decision limits which can detect milder forms, e.g. in $\mathrm{CH}$.

\section{Acknowledgements}

Institutional support was provided by the project DRO VFN64165 from the Ministry of Health of the Czech Republic, and by projects Progress Q26 and Q36 from Charles University.

\section{Conflict of Interests}

None declared

\section{REFERENCES}

1. Richter T, Nestler-Parr S, Babela R, Khan ZM, Tesoro T, Molsen E, et al. Rare disease terminology and definitions - a systematic global review: report of the ISPOR Rare Disease Special Interest Group. Value Health. 2015 Sep;18(6):906-14.

2. Council recommendation of 8 June 2009 on an action in the field of rare diseases. Off J Eur Union. 2009 Jul 3;52(C 151):7-10.

3. Government of the Czech Republic. National strategy for rare diseases 2010-2020. Prague: Government of the Czech Republic; 2010.

4. Votava F, Kozich V, Chrastina P, Peskova K, Adam T, Friedecky D, et al. Performance metrics of 5 years of newborn screening in the Czech Republic. Int J Neonatal Screen. 2016;2:69-70.

5. Wilson JM, Jungner YG. Principles and practise of mass screening for disease. Bol Oficina Sanit Panam. 1968;65(4):281-393. (In Spanish.)

6. Therrell BL, Padilla CD, Loeber JG, Kneisser I, Saadallah A, Borrajo GJ, et al. Current status of newborn screening worldwide: 2015. Semin Perinatol. 2015;39(3):171-87.

7. Methodical guideline for neonatal laboratory screening and follow-up care. Věstník MZ ČR. 2016;2016(6):2-11. (In Czech.)

8. Blehova B, Pazoutova M, Bloudkova D, Kroutilova O. Evaluation of screening for phenylketonuria after 6 years of existence of the laboratory. Cesk Pediatr. 1976;31(7):399-401. (In Czech.)

9. Hnikova O, Kracmar P, Zelenka Z, Philipiova O, Fabianova J, Skvor J, et al. Screening of congenital hypothyroidism in newborns in Bohemia and Moravia. Endocrinol Exp. 1989;23:117-23.

10. Votava F, Novotna D, Kracmar P, Vinohradska H, Stahlova-Hrabincova E, Vrzalova Z, et al. Lessons learned from 5 years of newborn screening for congenital adrenal hyperplasia in the Czech Republic: 17-hydroxyprogesterone, genotypes, and screening performance. Eur J Pediatr. 2012;171(6):935-40.

11. Sommerburg O, Krulisova V, Hammermann J, Lindner M, Stahl M, Muckenthaler M, et al. Comparison of different IRT-PAP protocols to screen newborns for cystic fibrosis in three central European populations. J Cyst Fibros. 2014;13(1):15-23. 
12. Chrastina P, Bartl J, Hornik P, et al. LCHAD deficiency - the most frequent fatty acid oxidation disorder in newborn screening in the Czech Republic. Cesk Slov Pediatr. 2009;64(4):175-6.

13. Buyukgebiz A. Newborn screening for congenital hypothyroidism. J Pediatr Endocrinol Metab. 2006;19(11):1291-8.

14. Pourfarzam M, Zadhoush F. Newborn Screening for inherited metabolic disorders; news and views. J Res Med Sci. 2013;18:801-8.

15. Léger J, Olivieri A, Donaldson M, Torresani T, Krude H, van Vliet G, et al. European Society for Paediatric Endocrinology consensus guidelines on screening, diagnosis, and management of congenital hypothyroidism. J Clin Endocrinol Metab. 2014;99(2):363-84.

16. Speiser PW, Azziz R, Baskin LS, Ghizzoni L, Hensle TW, Merke DP, et al. Congenital adrenal hyperplasia due to steroid 21-hydroxylase deficiency: an Endocrine Society clinical practice guideline. J Clin Endocrinol Metab. 2010;95(9):4133-60.

17. Mayell SJ, Munck A, Craig JV, Sermet I, Brownlee KG, Schwarz MJ, et al. A European consensus for the evaluation and management of infants with an equivocal diagnosis following newborn screening for cystic fibrosis. J Cyst Fibros. 2009;8(1):71-8.

18. van Spronsen FJ, van Wegberg AM, Ahring K, Bélanger-Quintana A, Blau N, Bosch AM, et al. Key European guidelines for the diagnosis and management of patients with phenylketonuria. Lancet Diabetes Endocrinol. 2017 Sep;5(9):743-56.

19. Leonard JV, Dezateux C. Newborn screening for medium chain acyl CoA dehydrogenase deficiency. Arch Dis Child. 2009;94(3):235-8.

20. The portal for rare diseases and orphan drugs [Internet]. Paris: Orphanet [cited 2018 May 25]. Available from: https://www.orpha.net/consor/ cgi-bin/Disease Search.php?lng=EN

21. Prevalence of rare diseases: bibliographic data. Orphanet Report Series, January 2018, Number 2 [Internet]. [cited 2018 May 30]. Available from: https://www.orpha.net/orphacom/cahiers/docs/GB/Prevalence_of_rare diseases by decreasing prevalence or cases.pdf.

22. Massie J, Curnow L, Gaffney L, Carlin J, Francis I. Declining prevalence of cystic fibrosis since the introduction of newborn screening. Arch Dis Child. 2010;95(7):531-3.
23. Vavrova V, Zemkova D, Skalicka V, Votava F. Problems in the diagnostics of cystic fibrosis - the need of newborn screening. Cesk Slov Pediatr. 2006;61(12):703-9.

24. Votava F, Kozich V, Chrastina P, Peskova K, Adam T, Friedecky D, et al. The results of expanded newborn screening in the Czech Republic. Cesk Slov Pediatr. 2014;69(2):77-86.

25. Loeber JG, Burgard P, Cornel MC, Rigter T, Weinreich SS, Rupp K, et al. Newborn screening programmes in Europe; arguments and efforts regarding harmonization. Part 1. From blood spot to screening result. J Inherit Metab Dis. 2012;35(3):603-11.

26. Frankova V, Votava F, Kozich V. Expansion of newborn screening for inherited metabolic disorders - ethical questions. Cesk Slov Pediatr. 2014;69(2):87-94

27. Dhillon K, Ho T, Rich P, Xu D, Lorey F, She J, et al. An automated method on analysis of blood steroids using liquid chromatography tandem mass spectrometry: application to population screening for congenital adrena hyperplasia in newborns. Clin Chim Acta. 2011;412(22-23):2076-84.

28. Ross LF, Clarke AJ. A historical and current review of newborn screening for neuromuscular disorders from around the world: lessons for the United States. Pediatr Neurol. 2017;77:12-22.

29. Burgard P, Rupp K, Linder M, Haege G, Rigter T, Weinreich SS, et al Newborn screening programmes in Europe; arguments and efforts regarding harmonization. Part 2 - From screening laboratory results to treatment, follow-up and quality assurance. J Inherit Metab Dis. 2012;35(4):613-25.

30. Svaton M, Sediva A, Mejstrikova E, et al. Differential diagnostics of immunodeficiency using antigen receptor excision circles in neonatal screening cards and in postnatal peripheral blood. Orv Hetil. 2012;153 Suppl 3:42.

Received June 28, 2018

Accepted in revised form January 15, 2019 